\title{
Preparation, characterization, and in vivo pharmacokinetics of thermosensitive in situ nasal gel of donepezil hydrochloride
}

\author{
FUGEN GU ${ }^{1, *}$ \\ HUIMIN FAN $^{2}$ \\ ZHIXIN CONG ${ }^{2}$ \\ SHUANG $\mathrm{LI}^{2}$ \\ YI WANG ${ }^{1}$ \\ CHUNZHI WU \\ ${ }^{1}$ Affiliated Hospital, Inner Mongolia \\ Medical University, Hohhot 010050 \\ China \\ ${ }^{2}$ School of Pharmacy, Inner Mongolia \\ Medical University, Hohhot 010110 \\ China
}

\begin{abstract}
Donepezil hydrochloride thermosensitive in situ gel for nasal delivery was prepared by using Poloxamer 407 and Poloxamer 188 as thermoreversible polymers, hydroxypropyl- $\beta$ -cyclodextrin and ethylparaben as permeation enhancer and preservative, respectively. The gelation temperature and time, $\mathrm{pH}$ value of the gel formulation were found to meet the requirements for nasal administration. The in vitro erosion and in vitro release tests exhibited obvious drug sustained release behavior. Meantime, main pharmacokinetic parameters such as $t_{\max } c_{\max }$ and $A U C$ in plasma as well as in brain were significantly different between the nasal gel formulation and intragastric drug solution in rats $(p<0.01)$. The relative bioavailability and drug targeting efficiency of the gel formulation were calculated to be 385.6 and $151.2 \%$, respectively. Thus, the drug gel formulation might be a potential new delivery system for treatment of Alzheimer's disease due to its higher bioavailability and better distribution to brain when compared to oral route.
\end{abstract}

Keywords: donepezil hydrochloride, thermosensitive in situ gel, nasal delivery, bioavailability, brain targeting

Alzheimer's disease (AD) is a central nervous system (CNS) disease characterized by loss of cognitive behavior, memory impairment, and disturbance in daily activities (1). The cholinergic deficit is thought to be one of the major pathological causes of this disease, thus some cholinesterase inhibitors such as risvastigmine, tacrine and donepezil hydrochloride (DPZ) have been clinically applied in order to improve the available levels of acetylcholine in brain tissues (2). DPZ is a specific and reversible AchE inhibitor and it also could produce neuroprotective effect (3). More importantly, the drug has fewer side effects than non-specific AchE inhibitors, so presently it has become a clinical first-line drug for treatment of mild to moderate AD in many countries (4).

As a CNS disease, treatment of AD by oral administration route is particularly challenging due to the existence of the blood-brain barrier (BBB) (5). So far, intranasal delivery has been thought as an important dosing route for some drugs with many advantages such as large mucosa surface area, rapid onset of action, avoidance of hepatic first pass effect, high

\footnotetext{
*Correspondence, e-mail: gfg1968@163.com
} 
bioavailability and especially circumvention of BBB (6). Furthermore, this administration route is non-invasive, of convenient application and good compliance. Therefore, intranasal dosing of drugs has been thought to be an ideal alternative to the control of AD (7).

Currently, the marketed dosage forms of DPZ are as oral tablets or capsules. Though this administration route is convenient for some of patients, the drug showed obvious gastrointestinal side effects and muscle convulsions (8). Moreover, the therapeutic efficacy of the DPZ oral preparations was not satisfactory due to the existence of BBB. Thus, in order to overcome the above shortcomings of oral route, some new dosage forms such as nanosuspension, microemulsion, liposomes and liposomal hydrogel of DPZ for nasal administration have been studied and furthermore the enhanced bioavailability as well as brain targeting characteristics of the above new nasal delivery systems also confirmed (9-12). However, the above novel delivery systems of DPZ for nasal route have many limitations such as complicated formulation and preparation process, inconvenient dosing, poor physicochemical stability, or short residence time in nasal cavity. Presently, drops and sprays are two main nasal preparations widely applied in clinical treatment, these traditional liquid formulations, however, are unfavorable because of short residence time and thus poor bioavailability. In recent years, in situ nasal gel has gained more attention for systemic drug delivery because of its advantages such as simple formulation, easy preparation process, better physiochemical stability, convenient use, accurate dose, long nasal residence time, high bioavailability, sustained drug release and potentially improved therapeutic efficacy (13). Thus, the aim of present research was to prepare a nasally administered thermosensitive in situ gel of DPZ and to further characterize its in vitro properties. In vivo pharmacokinetics, relative bioavailability, and especially brain targeting characteristics of the in situ gel formulation was also investigated in rats.

\section{EXPERIMENTAL}

\section{Materials}

Donepezil hydrochloride was bought from Hubei Hongxin Ruiyu Fine Chemical Co., Ltd. (China). Poloxamer 407 (P407) and poloxamer 188 (P188) were bought from BASF Co., Ltd. (China). Hydroxypropyl- $\beta$-cyclodextrin (HP- $\beta$-CD) was purchased from Shandong Fine Chemical Co., Ltd. (China). Ethylparaben was given by Aladdin Biochemical Technological Co., Ltd. (China). All other reagents were of analytical or chromatographic grades.

\section{Preparation of thermosensitive in situ gel}

Optimal formulation of DPZ thermosensitive in situ gel for nasal delivery was obtained from the results of our previous research and it was prepared by the cold method. Briefly, HP- $\beta$-CD $(3.5 \%, m / V)$ was first dissolved in suitable volume of distilled water by agitation at room temperature. The required amount of DPZ $(5.0 \%, \mathrm{~m} / \mathrm{V})$ and ethylparaben $(0.05 \%, m / V)$ were then dissolved successively in the above solution under ultrasonic condition. After cooling to room temperature, P407 $(19.6 \%, \mathrm{~m} / \mathrm{V})$ and P188 $(8.2 \%, \mathrm{~m} / \mathrm{V})$ was slowly added to the mixed solution and then fully dispersed by stirring. The dispersion was stored in cool condition overnight so that the poloxamer get completely dissolved and clear solution will be obtained. Finally, volume was adjusted to the requisite amount by using distilled water. 


\section{Gelation temperature and gelation time}

The gelation temperature of the in situ gel formulation was measured by the inverted-tube method as reported previously (14). $2 \mathrm{~mL}$ of the DPZ gel formulation was transferred to a $10 \mathrm{~mL}$ test tube which was sealed and immersed into a thermostat water bath. The temperature of the bath was increased from $20^{\circ} \mathrm{C}$ to $40{ }^{\circ} \mathrm{C}$ and a thermometer with a minimum scale of $0.1^{\circ} \mathrm{C}$ applied to accurately determine the temperature change. When the upper surface of the formulation solution wouldn't move if the test tube was turned 90 degrees, the temperature was thought as the gelation temperature. Similarly, $2 \mathrm{~mL}$ of the DPZ gel solution was transferred to a $10 \mathrm{~mL}$ volumetric flask and then heated to $20^{\circ} \mathrm{C}$ in a thermostat water bath. After which, the flask was placed into $32{ }^{\circ} \mathrm{C}$ thermostat water bath and the duration for all the liquid to change completely into immovable gel was immediately recorded with a stopwatch. The above time was identified as gelation time. The gelation temperature and gelation time were both determined in triplicate.

\section{pH of gel formulation}

The $\mathrm{pH}$ value of the gel solution was determined by using the $\mathrm{pH}$ meter, which was firstly calibrated prior to the test. Briefly, $1 \mathrm{~mL}$ of the prepared DPZ gel formulation was added into a $100 \mathrm{~mL}$ volumetric flask, then dilute with freshly distilled water to volume and mix. The $\mathrm{pH}$ value of the above solution was determined in triplicate.

\section{In vitro erosion and release studies}

In vitro corrosion and release of DPZ from the gel formulation were investigated simultaneously by the membraneless method (15). Briefly, $10 \mathrm{~g}$ of DPZ gel solution was transferred into a pre-weighted test tube, which was then placed in water bath $(34 \pm 0.5$ ${ }^{\circ} \mathrm{C}$ ) and maintained for $5 \mathrm{~min}$. After completion of sol-gel transition, $1.0 \mathrm{~mL}$ of $\mathrm{pH} 6.4$ phosphate buffer pre-warmed at $34 \pm 0.5^{\circ} \mathrm{C}$, was gently added over the surface of the gel and the test tube was immediately shaken at $50 \mathrm{r} \mathrm{min}^{-1}$ in a thermostat oscillator maintained at $34{ }^{\circ} \mathrm{C}$. After dumping the medium at specified time interval, the test tube was cleared, weighted and replaced with the same volume and temperature of fresh phosphate buffer. The DPZ in the samples was determined by reported HPLC method with minor modification (16). The analysis was performed on a $\mathrm{C}_{18}$ column $(250 \times 4.6 \mathrm{~mm}, 5.0$ $\mu \mathrm{m})$ at the column temperature of $30^{\circ} \mathrm{C}$. The mobile phase consisted of methanol and 20 mmol L ${ }^{-1}$ ammonium acetate solution (containing $0.05 \%$ formic acid) $(55: 45, V / V)$ and the

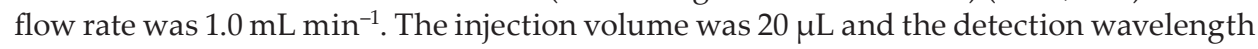
was set at $271 \mathrm{~nm}$.

Moreover, in vitro release studies were also performed to observe the DPZ sustained release from the in situ gel as compared with the drug solution by dialysis diffusion technique. Briefly, $2 \mathrm{~mL}$ of the in situ gel as well as drug solution equivalent to $100 \mathrm{mg}$ of DPZ was placed in a dialysis bag (MWCO, $3500 \mathrm{Da})$ and then sealed at both ends. After which, the dialysis bags were placed in $500 \mathrm{~mL}$ of $\mathrm{pH} 6.4$ phosphate buffer thermostatically maintained at $37 \pm 0.5{ }^{\circ} \mathrm{C}$, and immediately stirred at speed of $100 \mathrm{rpm}$. At specific time intervals $(5,15,30,60,120,180,240,360$ and $480 \mathrm{~min}), 2 \mathrm{~mL}$ of samples was withdrawn and filtered through a $0.45 \mu \mathrm{m}$ syringe filter. The resultant filtrates were analyzed by above HPLC method. 


\section{Pharmacokinetics and brain targeting studies}

Sprague-Dawley male rats weighing 250-320 g were selected for the study and an experimental protocol was approved by our Institutional Ethical Committee for animal and human tests (Grant No.YKD2018068). The rats were randomly divided into control and test groups. The control group received DPZ solution at a dose of $10 \mathrm{mg} \mathrm{kg}^{-1}$ through intragastric (i.g.) route. The test group rats were firstly anesthetized with ether, after which, the trachea was cannulated with a polyethylene tube to stop nasal respiration. Meanwhile, an incision was made in the esophagus, after which, a length of polyvinyl chloride (PVC) pipe was inserted into it, the DPZ gel solution was then given at the same dose with a microliter syringe stretching into the back of the nasal cavity via the PVC pipe. The PVC pipe was subsequently pulled out and the esophagus ligated to prevent peroral absorption. For the control group, five rats per time point were sacrificed at 5, 15, 30, 45, 60, 90, 120, 180, 240, 360, $480 \mathrm{~min}$ following i.g. administration of the drug solution. Blood was collected and placed into heparinized PE tubes, immediately centrifuged at 10,000 rpm for $10 \mathrm{~min}$ and plasma separated. Meantime, the brain was isolated, rinsed with normal saline and weighed followed by homogenization using glass homogenizer with suitable amount of saline in ice-water bath. Homogenized brain tissue was centrifuged and the brain supernatant separated. For the test group, the same number of animals each time point were also sacrificed at 3, 5, 10, 20, 30, 45, 60, 90, 120, 180, 240 min after nasal administration of the DPZ in situ gel. The blood and brain tissues were pretreated as described above for i.g. dosing. Finally, the separated plasma and brain supernatant were stored at $-20^{\circ} \mathrm{C}$ until being assayed.

\section{Plasma and brain sample analysis}

In order to further improve the analytical sensitivity, the pretreatment process of blood and brain samples involving concentrating the sample and extracting the drug, was completed prior to analysis. For analysis of drug in plasma, $20 \mu \mathrm{L}$ of $0.1 \mathrm{~mol} \mathrm{~L}^{-1} \mathrm{NaOH}$ solution and $300 \mu \mathrm{L}$ of $n$-hexane/dichloromethane/ethyl acetate mixture (45:40:15, $V / V / V)$ were successively added to $100 \mu \mathrm{L}$ plasma sample and vortex-mixed for $3 \mathrm{~min}$. After which, the mixture was centrifuged at 10,000 rpm for $10 \mathrm{~min}$, and then the supernatant was transferred to a clean PE tube and evaporated to dryness under a nitrogen gas stream in a $40{ }^{\circ} \mathrm{C}$ water bath. The residue was then reconstituted in $400 \mu \mathrm{L}$ methanol, and after centrifugation, $20 \mu \mathrm{L}$ of the supernatant was injected for HPLC analysis. For drug analysis in brain homogenates, $20 \mu \mathrm{L}$ of $0.1 \mathrm{~mol} \mathrm{~L}^{-1} \mathrm{NaOH}$ solution and $300 \mu \mathrm{L}$ of methyl t-butyl ether were successively added to $100 \mu \mathrm{L}$ of brain supernatant sample and vortex-mixed for $3 \mathrm{~min}$. The mixture was also centrifuged at 10,000 rpm for $5 \mathrm{~min}$, and then $200 \mu \mathrm{L}$ of supernatant was transferred to a PE tube and evaporated to dryness. The residue were treated as described above for plasma samples and assayed for DPZ content. Finally, DPZ content in plasma and brain samples was determined according to a modified HPLC method (17). Chromatographic separation was achieved with a Inert Sustain $C_{18}$ column $(150 \mathrm{~mm} \times 4.6 \mathrm{~mm}, 5 \mu \mathrm{m})$. The mobile phase consisted of $0.01 \mathrm{~mol} \mathrm{~L}^{-1}$ sodium dihydrogen phosphate solution and acetonitrile in a ratio of 73:27 $(V / V)$. The mobile phase was set at a flow rate of $1.2 \mathrm{~mL}$ $\mathrm{min}^{-1}$. The eluate was monitored at $390 \mathrm{~nm}$ with an excitation wavelength of $325 \mathrm{~nm}$. The concentration of DPZ in plasma or brain samples was estimated according to each calibration curve over the concentration range from 10.0 to $800.0 \mathrm{ng} \mathrm{mL}^{-1}$. 
Pharmacokinetic analysis was performed by a model-independent method and the mean concentrations of DPZ in plasma and brain samples were plotted against time. The peak plasma or brain concentrations $\left(c_{\max }\right)$ as well as the time to reach these peaks $\left(t_{\max }\right)$ were read as raw data. The area under the plasma or brain concentration-time curve $\left(A U C_{0-t}\right)$ from time zero to the time of the last measured concentration $\left(c_{t}\right)$ was calculated by means of the linear trapezoidal rule. The bioavailability $\left(F_{\mathrm{r}}\right)$ of the DPZ gel formulation relative to i.g. drug solution was calculated. Drug targeting efficiency (DTE \%) of the in situ gel after nasal administration versus i.g. drug solution was estimated using the following equation(18):

$$
\operatorname{DTE}(\%)=\left(A U C_{\text {brain 0-t }} / A U C_{\text {plasma 0-t }}\right)_{\text {i.n. }} /\left(A U C_{\text {brain 0-t }} / A U C_{\text {plasma 0-t }}\right)_{i . g .} \times 100 \%
$$

where $A U C_{\text {brain }}$ and $A U C_{\text {plasma }}$ is the area under the brain tissue concentration-time and plasma concentration-time curves after i.n. and i.g. administration, respectively.

\section{RESULTS AND DISCUSSION}

\section{Preparation of in situ gel}

Comprehensively taking into consideration the limitation of volume of human nasal cavity and clinical doses of oral preparations, the drug concentration of the DPZ gel formulation was established to be $5.0 \%(\mathrm{~m} / \mathrm{V})$. As main excipients in the gel formulation, the ideal concentration of P407 as well as P188 was found to be $19.6 \%(\mathrm{~m} / \mathrm{V})$ and $8.2 \%(\mathrm{~m} / \mathrm{V})$, respectively, which was confirmed by our previous research (19). Owing to their higher aqueous solubility and better safety, several $\beta$-CD derivatives such as HP- $\beta-C D$, SBE- $\beta-C D$ and DM$-\beta-C D$ have been widely applied as transmucous absorption enhancers in nasal formulations. The ex vivo sheep nasal mucosa penetration test performed by our research group has confirmed that $3.5 \%(\mathrm{~m} / \mathrm{V}) \mathrm{HP}-\beta$-CD showed the best permeability enhancing effect among the above cyclodextrin enhancers (19). In addition, based on the concentration of ethylparaben often applied in oral and ophthalmic preparations, $0.05 \%(\mathrm{~m} / \mathrm{V})$ of the preservative was used in the drug gel formulation. Finally, it needs to be emphasized that in the preparation process of the DPZ in situ gel solution, the above HP- $\beta$-CD used in the formulation, could also increase the solubility of the poor water-soluble ethylparaben by complexation reactions, which made it easier to dissolve the preservative in the HP- $\beta$-CD solution.

The polymeters P407 and P188 are the most popular thermosensitive gelling agents used for the topical applications through the nasal, ocular and cutaneous routes and also have lower adverse effect on tissues compared with other stimuli-responsive gelling systems (20). Furthermore, $0.05 \%$ ethylparaben is often used in oral and ophthalmic preparations and HP- $\beta$-CD used as additive in the intravenous injections. Based on the above analysis, it could be anticipated that the DPZ gel formulation may have lower toxicity and higher safety in vivo. Certainly, a study on the toxicity and human safety of the formulation still needs to be further investigated.

\section{Gelation temperature and gelation time}

Gelation temperature and gelation time are two important quality indexes for the DPZ thermoreversible in situ gel since they have been proved to be closely correlated with 
the storage condition, convenience of administration, nasal residence time and in vivo drug bioavailability of in situ nasal gel. Gelation temperature value of the DPZ gel formulation was found to be approximately $32.5^{\circ} \mathrm{C}$ within the nasal physiological temperature range 29-34 ${ }^{\circ} \mathrm{C}$, thus suitable for thermosensitive nasal gel formulations. Furthermore, gelation time of the gel formulation was determined to be only about $40 \mathrm{~s}$, suggesting rapid formation of immovable gel after its contact with nasal mucosa. In short, the above gelation temperature and gelation time are generally considered as optimal, which might reduce or even avoid loss of drug due to transportation of the formulation to the nasopharynx and then to the gastrointestinal tract.

\section{pH of formulation}

The $\mathrm{pH}$ of the gel formulation was determined after suitable dilution because of the following reasons. Firstly, the gel formulation has relatively higher viscosity, so it was not easy to obtain a stable readings of $\mathrm{pH}$ meter in testing process. Secondly, considering the high rate of nasal mucus turnover, for example, resting-state daily production, $75-150 \mathrm{~mL}$, thus the dose volume of the gel solution may be diluted in nasal cavity by at least 100 times following nasal dosing. Finally, according to the chemical structures of the drug and additives in the gel formulation, $\mathrm{pH}$ of the gel formulation theoretically seems to be nearly neutral. Therefore, diluting the gel sample by distilled water may have insignificant effect on the $\mathrm{pH}$ of the gel solution.

Previous research performed by our group has confirmed that DPZ had high oil-water partition coefficient (lipophilicity) and better chemical stability in an approximately $\mathrm{pH} 7.0$ aqueous media, and furthermore ethylparaben also possesses relatively good bacteriostatic effect in the $\mathrm{pH}$ range 4.0-8.0. Additionally, human nasal physiological $\mathrm{pH}$ range was reported to be within 5.5-6.5 and it has been proved that the nasal mucosa can tolerate $\mathrm{pH}$ range of 3-10 (21) and greater deviation from nasal physiological $\mathrm{pH}$ could lead to severe irritancy or damage to mucosa. Thus, taking the above factors into consideration, neutral $\mathrm{pH}$ value of the gel formulation seemed to be appropriate. Since the $\mathrm{pH}$ value of the prepared gel formulation was determined to be about 7.0, it is unnecessary to adjust the $\mathrm{pH}$ value of the gel solution in the final preparation process of the DPZ in situ gel.

\section{In vitro erosion and release studies}

The membraneless model was applied to investigate the gel corrosion and DPZ release from the thermosensitive gel formulation in our research as this model is quite similar to the in vivo condition. The concentrations of DPZ in the release media were calculated from the standard calibration curve $\left(Y=69970 c-127740, R^{2}=0.999\right.$, concentration range of $0.8-$ $200.0 \mu \mathrm{g} \mathrm{mL}^{-1}$ ) of DPZ and the cumulative release of drug as well as gel corrosion was plotted against time. As shown in Fig. 1, the in vitro cumulative erosion and cumulative drug release were both approximately $90 \%$ in $45 \mathrm{~min}$. The drug release from the gel formulation seemed rapid, which might be mainly attributable to the use of big volume of release media in the corrosion and drug release study, for example, $1.0 \mathrm{~mL}$ of phosphate buffer each time, which far larger than the actual volume of nasal mucus, was added over the surface of the gel. Furthermore, the sample-containing test tube was shaken at higher speed, resulting in the faster gel corrosion. The in vitro erosion and in vitro drug release kinetics of the DPZ in situ gel were both fitted to the zero order model, and moreover, there 


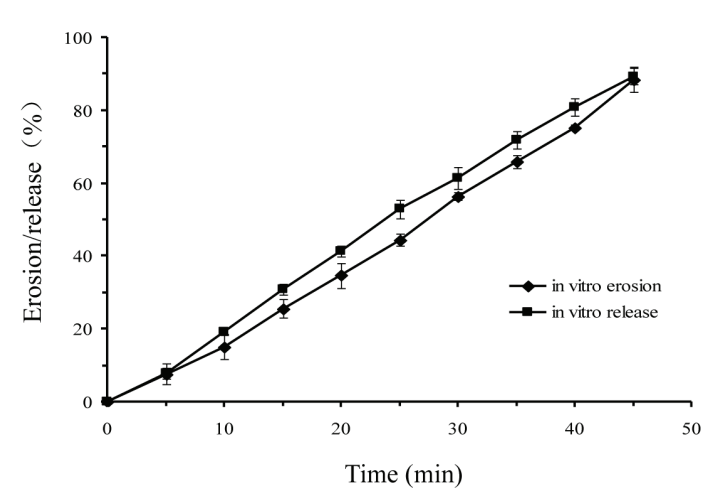

Fig. 1. The in vitro erosion and in vitro drug release kinetics of DPZ in situ gel with membraneless method. Data are represented as mean $\pm \mathrm{SD}(n=3)$.

was a good correlation relationship between them $\left(R^{2}=0.9918\right)$, which indicated that release of DPZ from the gel formulation was mainly controlled by gel corrosion.

Meanwhile, the DPZ sustained release from the gel formulation was also investigated by dialysis membrane diffusion method. An obvious drug sustained-release characteristics was observed in Fig. 2, for example, the cumulative release of DPZ from the gel formulation was only less than $80 \%$ till $480 \mathrm{~min}$, and $85 \%$ of the drug, however, was released from the drug solution within $120 \mathrm{~min}$. To explain the mechanism of DPZ release from the gel formulation, the in vitro release data of the drug were kinetically evaluated by using mathematical models. The order of the correlation coefficient $\left(R^{2}\right)$ values of model fitting was found to be Weibull model $>$ Riger-Pappas model $>$ Higuchi model $>$ First-order model $>$ Zero-order model. The best fitted model was the Weibull model $\left(R^{2}=0.9947\right)$, suggesting that the drug release from the gel formulation was controlled by drug diffusion as well as gel corrosion, probably due to the use of semi-permeable membrane in the in vitro release test.

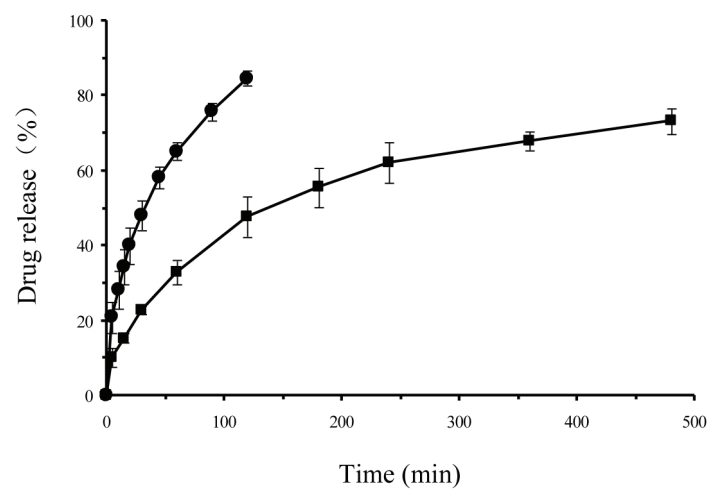

Fig. 2. The in vitro release profiles of DPZ in situ gel (squares) as well as the drug solution (circles) with dialysis diffusion method. Data are represented as mean $\pm \mathrm{SD}(n=3)$. 


\section{In vivo pharmacokinetics and brain targeting characteristics}

For the test group rats, after nasal dosing of the DPZ gel solution via a microliter syringe, the esophagus was ligated in order to prevent the gel formulation from entering GI tract and further avoid peroral absorption. As a result, the administered dose of the drug for nasal absorption might be accurate. Moreover, since nasal solution has shorter residence time relative to nasal in situ gel, a comparison with nasal DPZ solution has not been implemented in our research.

DPZ concentrations in plasma and brain after nasal administration of the in situ gel and i.g. drug solution to rats were calculated from each calibration curve equation. For the quantitative analysis of DPZ in plasma samples, the obtained calibration equation $(A=$ $\left.111.14 c+10000, R^{2}=0.9982\right)$ showed good linearity over the concentration range of $10.0-$ $800.0 \mathrm{ng} \mathrm{mL}^{-1}$. Accuracy of DPZ determination was found to be in range of $96.94-103.50 \%$. Intra-day and inter-day precision values were both lower than $5.0 \%$. Mean extraction recovery of DPZ in rat plasma at 50.0, 200.0, $600.0 \mathrm{ng} \mathrm{mL}^{-1}$ were $97.92,104.46$ and $98.40 \%$, respectively. Additionally, as for the analysis of drug in brain samples, the linearity range was also from 10.0 to $800.0 \mathrm{ng} \mathrm{mL}^{-1}$ with correlation coefficient of 0.9994 and the standard curve equation was $A=105 c+4888$.2. Intra-day and inter-day precision values were both less than $2.0 \%$. Accuracy of DPZ determination was found to be in range of $96.29-104.28 \%$. Mean extraction recovery of DPZ in rat brain at 50.0, 200.0, 600.0 $\mathrm{ng} \mathrm{mL}^{-1}$ was between 97.12 and $104.43 \%$. In short, the above established methods were suitable for the determination of DPZ in plasma as well as brain tissues.

The mean plasma concentration-time curves of DPZ after nasal administration of the gel formulation and i.g. drug solution at dose of $10 \mathrm{mg} \mathrm{kg}^{-1}$ and obtained pharmacokinetic parameters are shown in Fig. 3 and Table I, respectively. It could be found that the drug following nasal dosing was more rapidly absorbed from the in situ gel than from the drug solution after i.g. administration. The obtained $c_{\max }$ of the drug in situ gel was $4113.41 \mathrm{ng}$ $\mathrm{mL}^{-1}$, which was approximately 15 times higher than that for the i.g. drug solution. Meantime, $t_{\max }$ for the nasal route was found to be only $10 \mathrm{~min}$, which was much less than 55

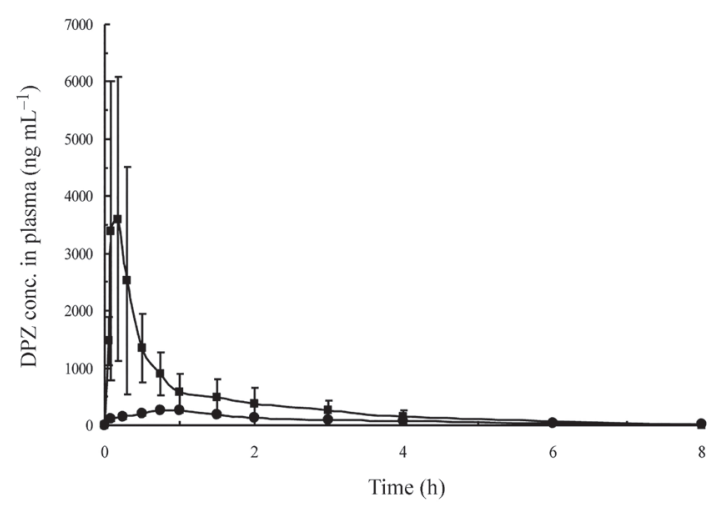

Fig. 3. Mean plasma concentration-time curves of DPZ after nasal administration of in situ gel (squares) as well as intragastric drug solution (circles) at the dose of $10 \mathrm{mg} \mathrm{kg}^{-1}$ to rats. Data are represented as mean $\pm \mathrm{SD}(n=5)$. 
min for i.g. route. Furthermore, when the $A U C$ s of both the dosing route were compared, the DPZ gel formulation had higher $A U C$ value, for instance, $A U C_{0-\infty}$ for nasal route was up to $3010.10 \mathrm{ng} \mathrm{h} \mathrm{mL}^{-1}, A U C_{0-\infty}$ for the latter route, however, was only $780.66 \mathrm{ng} \mathrm{h} \mathrm{mL}^{-1}$. Therefore, there was significant difference in $t_{\max }, c_{\max }$ and $A U C$ values between the nasal gel formulation and the i.g. drug solution $(p<0.01)$. The relative bioavailability of the DPZ gel formulation was calculated to be up to $385.6 \%$, suggesting significantly enhanced bioavailability as compared to i.g. route. Compared to i.g. drug solution, the rapid absorption rate and significantly improved bioavailability of the DPZ in situ gel for nasal delivery in rats may be attributed to avoidance of hepatic first pass metabolism and enhanced drug permeability through the nasal mucosa by HP- $\beta$-CD. Moreover, the prolonged nasal residence time of the gel formulation also could result in better bioavailability. It also needs to be pointed out that the above results were in agreement with previous report by Al Asmari et al. (11), which proved that nasally administered liposomal formulation of DPZ showed significantly increased bioavailability in rats. The gel formulation in our study, however, have more advantages such as simple formulation, easy preparation process, better physiochemical stability, long nasal residence time over the liposomal formulation. Furthermore, the DPZ in situ gel could tightly adhere to the nasal mucosa and thereby prolonged the release and contact time. Thus, the use of the gel formulation resulted in higher systemic bioavailability as compared with the liposomal formulation.

As depicted in Fig. 4 and Table II, the peak concentration of DPZ in brain $\left(c_{\max }\right)$ and the time to reach it $\left(t_{\max }\right)$ following nasal administration of the in situ gel to rats were found to be $11218.34 \mathrm{ng} \mathrm{mL}^{-1}$ and $14 \mathrm{~min}$, respectively, for the i.g. drug solution, however, the values of $c_{\max }$ and $t_{\max }$ were $757.85 \mathrm{ng} \mathrm{mL} \mathrm{mL}^{-1}$ and $90 \mathrm{~min}$, respectively, suggesting rapid and greater transport of DPZ into the brain $(p<0.01)$. Moreover, it was observed that DPZ concentration in the brain after intranasal in situ gel was far higher at almost all time points than after the i.g. drug solution. The $A U C_{0-t}$ of DPZ in brain for the gel formulation was found to be more than five times greater compared with i.g. drug solution $(p<0.01)$. The above results demonstrated better bioavailability of DPZ in brain after nasal administration of the in situ gel compared to i.g. drug solution. Meanwhile, the DTE value was calculated to be $151.16 \%$, which was greater than 100 , also suggesting superior drug delivery to the brain with the in situ gel. Our findings were similar to the results of the previous studies showing increased brain bioavailability of liposomal DPZ and the drug nanosuspension following intranasal dosing in rats $(10,11)$. Moreover, the brain targeting of nasally administered

Table I. Main pharmacokinetic parameters of donepezil hydrochloride in plasma after nasal adminstration of the in situ gel to rats using intragastric drug solution as control. Data are represented as mean $\pm S D(\mathrm{n}=5)$.

\begin{tabular}{ccc}
\hline Parameter & Drug solution (i.g.) & In situ gel (i.n.) \\
\hline$t_{\max }(\min )$ & $55 \pm 7.75$ & $10 \pm 5.48^{\mathrm{a}}$ \\
$c_{\max }\left(\mathrm{ng} \mathrm{mL}^{-1}\right)$ & $269.35 \pm 27.06$ & $4113.41 \pm 2729.68^{\mathrm{a}}$ \\
$A U C_{0-\mathrm{t}}\left(\mathrm{ng} \mathrm{h} \mathrm{mL}^{-1}\right)$ & $735.68 \pm 108.41$ & $2718.25 \pm 1373.59^{\mathrm{a}}$ \\
$A U C_{0-\infty}\left(\mathrm{ng} \mathrm{h} \mathrm{mL}^{-1}\right)$ & $780.66 \pm 127.71$ & $3010.10 \pm 1705.56^{\mathrm{a}}$ \\
$F_{\mathrm{r}}$ & $385.58 \%$ \\
\hline
\end{tabular}

${ }^{a} p<0.01$, compared to the control; $F_{\mathrm{r}}$ - bioavailability relative to intragastric drug solution. 


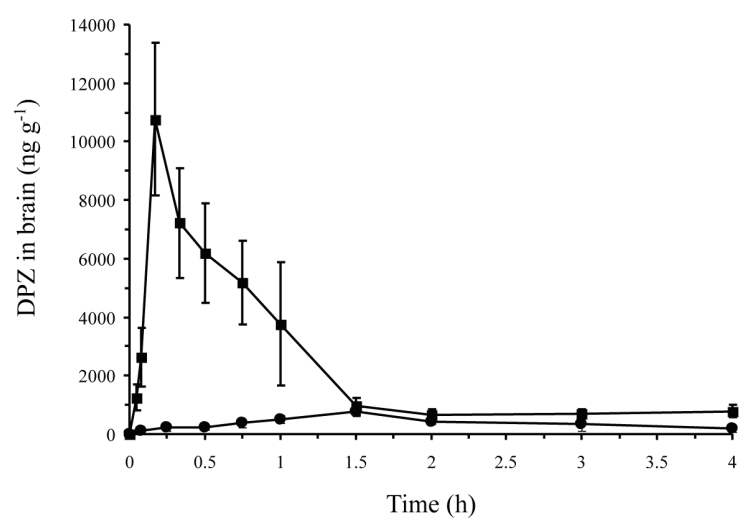

Fig. 4. Mean brain concentration-time curves of DPZ after nasal administration of the in situ gel (squares) and intragastric drug solution (circles) at the dose of $10 \mathrm{mg} \mathrm{kg}^{-1}$ to rats. Data are represented as mean $\pm \mathrm{SD}(n=5)$.

DPZ was also confirmed by the pharmacokinetic study accomplished in rabbits, which indicated that intranasal delivery of the drug through liposomal hydrogel increase the brain intake of the drug compared to the oral route (12). There can be several reasons for the increased brain delivery for the DPZ gel formulation. Firstly, one possible reason may be that the better bioavailability and the greater plasma concentrations of DPZ with the gel formulation could result in more easily diffusing of the drug across the BBB due to the higher concentration gradient from the systemic circulation to the brain. In this respect, $\mathrm{HP}-\beta$-CD might play an indirect role in improving brain bioavailability of DPZ gel formulation since it could promote the absorption of the drug across nasal mucosa and therefore significantly improved the plasma concentration and the bioavailability of the drug in plasma, which eventually led to enhanced transport of the drug from the blood system into the brain. Another possible reason may be that P188 in the gel formulation probably acted as absorption enhancers and furthermore inhibited the efflux system, especially $P$-glycoprotein, also increasing the transport across BBB. Finally, the preferential delivery into brain of the in situ gel may result from the direct transport of DPZ into brain via the olfactory or trigeminal nerves.

Table II. Main pharmacokinetic parameters of donepezil hydrochloride in brain after nasal administration of the in situ gel to rats using intragastric drug solution as control ${ }^{\text {a }}$

\begin{tabular}{|c|c|c|}
\hline Parameters & Drug solution (i.g.) & In situ gel (i.n.) \\
\hline$t_{\max }(\min )$ & $90 \pm 0.00$ & $14 \pm 5.48^{\mathrm{b}}$ \\
\hline$c_{\max }\left(\operatorname{ng~g}^{-1}\right)$ & $757.85 \pm 137.82$ & $11218.34 \pm 1953.25^{b}$ \\
\hline$A U C_{0-\mathrm{t}}\left(\mathrm{ng} \mathrm{h} \mathrm{g}^{-1}\right)$ & $1581.32 \pm 353.56$ & $8832.22 \pm 1397.13^{b}$ \\
\hline DTE (\%) & & \\
\hline
\end{tabular}

${ }^{\mathrm{a}}$ Data are represented as mean $\pm \mathrm{SD}(n=5) .{ }^{\mathrm{b}} p<0.01$, compared to the control; DTE - drug targeting efficiency. 
Due to the lack of nasal spray pumps and specific instruments, the aerosol performance of the gel formulation related to the several important parameters including droplet size, spray pattern, deposition pattern, etc. was not accomplished in this study, thus our research group wishes to complete it in near future.

\section{CONCLUSIONS}

DPZ thermosensitive in situ gel for nasal delivery was prepared by using P407 and P188 as main gel materials. The gel formulation exhibited ideal in vitro properties, such as suitable gelation temperature, short gelation time, approximately neutral $\mathrm{pH}$, obvious sustained drug release. Moreover, pharmacokinetic studies confirmed that the bioavailability of the drug in situ gel formulation after nasal administration to rats was significantly increased compared to the drug solution after i.g. dosing. Brain targeting investigation also proved the remarkably enhanced transport of DPZ into the brain by intranasal in situ gel formulation. Thus, it is anticipated that the DPZ thermosensitive nasal in situ gel will be an ideal novel delivery system for the treatment of AD's disease due to lots of advantages over the other new delivery systems of the drug such as simple formulation, easy preparation process, convenient dosing, rapid onset of action, decreased dosing frequency, high bioavailability, significant brain targeting and potentially improved therapeutic efficacy. Finally, the pharmacokinetics and brain targeting of the DPZ in situ gel formulation in humans as well as its toxicity or irritation to animal or human nasal mucosa are unknown, so a further research is also necessary and furthermore very important.

Acknowledgements. - This study was supported by Grants from the Natural Scientific Foundation of Inner Mongolia (Grant No. 2018MS 08107).

\section{REFERENCES}

1. C. A. Lane, J. Hardy and J. M. Schott, Alzheimer's disease, Eur. J. Neurol. 25 (2018) 59-70; https:// doi.org/10.1111/ene.13439

2. L. A. Craig, N. S. Hong and R. J. McDonald, Revisiting the cholinergic hypothesis in the development of Alzheimer's disease, Neurosci. Biobehav. Rev. 35 (2011) 1397-1409; https://doi.org/ 10.1016/j. neubiorev.2011.03.001

3. T. Maurice, Protection by sigma-1 receptor agonists is synergic with donepezil, but not with memantine, in a mouse model of amyloid-induced memory impairments, Behav. Brain Res. 296 (2016) 270-278; https://doi.org/ 10.1016/j.bbr.2015.09.020

4. K. Meguro, M. Kasai, M. Meguro, H. Ishii and S. Yamaguchi, Donepezil and life expectancy in Alzheimer's disease: A retrospective analysis in the Tajiri Project, BMC Neurol. 14 (2014) 83; https:// doi.org/ 10.1186/1471-2377-14-83

5. E. Garcia-Garcia, K. Andrieux, S. Gil and P. Couvreur, Colloidal carriers and blood-brain barrier (BBB) translocation: a way to deliver drugs to the brain? Int. J. Pharm. 298 (2005) 274-292; https:// doi.org/10.1016/j.ijpharm.2005.03.031

6. C. V. Pardeshi and V. S. Belgamwar, Direct nose to brain drug delivery via integrated nerve pathways bypassing the blood-brain barrier: an excellent platform for brain targeting, Expert Opin. Drug Del. 10 (2013) 957-972; https://doi.org/10.1517/17425247.2013.790887

7. M. Agrawal, S. Saraf, S. Saraf, S. G. Antimisiaris, M. B. Chougule, S. A. Shoyele and A. Alexander, Nose-to-brain drug delivery: An update on clinical challenges and progress towards approval of 
anti-Alzheimer drugs, J. Control. Release 281 (2018) 139-177; https://doi.org/ 10.1016/j.jconrel.2018.05.011

8. C. Christodoulou, P. Melville, W. F. Scherl, W. S. Macallister, L. E. Elkins and L. B. Krupp, Effects of donepezil on memory and cognition in multiple sclerosis, J. Neurol. Sci. 245 (2006) 127-136; https://doi.org/10.1016/j.jns.2005.08.021

9. L. C. Espinoza, M. Vacacela, B. Clares, M. L. Garcia, M. J. Fabrega and A. C. Calpena, Development of a nasal donepezil-loaded microemulsion for the treatment of Alzheimer's disease: in vitro and ex vivo characterization, CNS Neuro. Disord. Drug Targets 17 (2018) 43-53; https://doi.org/10.2174/1 871527317666180104122347

10. Bhavna, Shadab Md, M. Ali, R. Ali, A. Bhatnagar, S. Baboota and J. Ali, Donepezil nanosuspension intended for nose to brain targeting: In vitro and in vivo safety evaluation, Int. J. Biol. Macromol. 67 (2014) 418-425; https://doi.org/ 10.1016/j.ijbiomac.2014.03.022

11. A. K. Al Asmari, Z. Ullah, M. Tariq and A. Fatani, Preparation, characterization, and in vivo evaluation of intranasally administered liposomal formulation of donepezil, Drug Des. Devel. Ther. 10 (2016) 205-215; https://doi.org/ 10.2147/DDDT.S93937

12. S. Al Harthi, S. E. Alavi, M. A. Radwan, M. M. El Khatib and I. A. Al Sarra, Nasal delivery of donepezil HCl-loaded hydrogels for the treatment of Alzheimer's disease, Sci. Rep. 9 (2019) 9563; https://doi.org/10.1038/s41598-019-46032-y

13. C. Karavasili and D. G. Fatouros, Smart materials: in situ gel-forming systems for nasal delivery, Drug Discov. Today 21 (2016) 157-166; https://doi.org/10.1016/j.drudis.2015.10.016

14. J. C. Gilbert, J. Hadgraft, A. Bye and L. G. Brookes, Drug release from Pluronic F-127 gels, Int. J. Pharm. 32 (1986) 223-228; https://doi.org/ 10.1016/0378-5173(86)90182-1

15. L. E. Jabarian, M. R. Rouini, F. Atyabi, A. Foroumadi, S. M. Nassiri and R. Dinarvand, In vitro and in vivo evaluation of an in situ gel forming system for the delivery of PEGylated octreotide, Eur. J. Pharm. Sci. 48 (2013) 87-96; https://doi.org/ 10.1016/j.ejps.2012.09.017

16. Y. C. Li, H. Xin and L. Gao, To establish an HPLC-ESI-SIM method for the determination of donepezil in human plasma, Chin. J. Aesthetic Med. 21 (2012) 24-26; https://doi.org/10.15909/j.cnki.cn611347/r.2012.16.451

17. M. Valis, J. Masopust, O. Vysata, J. Hort, R. Dolezal, J. Tomek, J. Misik, K. Kuca and J. Z. Karasova, Concentration of donepezil in the cerebrospinal fluid of AD Patients: Evaluation of dosage sufficiency in standard treatment strategy, Neurotox. Res. 31 (2017) 162-168; https://doi.org/ 10.1007/ s12640-016-9672-y

18. U. C. Galgatte, A. B. Kumbhar and P. D. Chaudhari, Development of in situ gel for nasal delivery: design, optimization, in vitro and in vivo evaluation, Drug Deliv. 21 (2014) 62-73; https://doi.org/10 $.3109 / 10717544.2013 .849778$

19. H. M. Fan, Z. X. Cong and F. G. Gu, Preparation and in vitro properties of thermosensitive in situ nasal gel of donepezil hydrochloride, Centr. South Pharm. 16 (2018) 1194-1199; https://doi. org/10.7539/j.issn.1672-2981.2018.09.003 (in Chinese only)

20. Q. Wang, Z. Zuo, C. K. C. Cheung and S. S. Y. Leung, Updates on thermosensitive hydrogel for nasal, ocular and cutaneous delivery, Int. J. Pharm. 559 (2019) 86-101; https://doi.org/10.1016/j. ijpharm.2019.01.030 10.1016/j.ijpharm.2019.01.030

21. C. R. Behl, H. K. Pimplaskar, A. P. Sileno, J. deMeireles and V. D. Romeo, Effects of physicochemical properties and other factors on systemic nasal drug delivery, Adv. Drug Deliv. Rev. 29 (1998) 89-116; https://doi.org/10.1016/s0169-409x(97)00063-x 